\title{
Validación externa del modelo HUSI para la predicción de coledocolitiasis en pacientes con colelitiasis-colecistitis
}

\author{
External validation of the HUSI model for prediction of choledocholithiasis \\ in patients with cholelithiasis-cholecystitis
}

\author{
Julián Henao1, Stevenson Orlando Londoño1, Sebastián Camilo Monsalve1, \\ Víctor Manuel Quintero², Álvaro Sanabria2,3
}

1 Médico, residente de Cirugía General, Universidad de Antioquia, Medellín, Colombia

2 Médico, profesor de Cirugía General, Universidad de Antioquia, Hospital Pablo Tobón Uribe, Medellín, Colombia

3 Fundación Colombiana de Cancerología, Clínica Vida, Medellín, Colombia

Primer puesto en el concurso Simposio Nacional del Residente Quirúrgico, 43 Congreso Nacional "Avances en Cirugía", 2017, Medellín, Colombia

\section{Resumen}

Introducción. La probabilidad de que los pacientes con colelitiasis cursen con coledocolitiasis y determinar si realmente el cálculo se encuentra en la vía biliar, siguen siendo un reto para el cirujano. La toma de decisiones puede generar costos elevados, por lo que se requiere evaluar con exactitud la probabilidad de cálculos en la vía biliar y la utilidad de las escalas de probabilidad.

Objetivo. Validar externamente el modelo de predicción de coledocolitiasis desarrollado por Domínguez, et al., en el Hospital Universitario de San Ignacio, haciendo una evaluación preoperatoria de la coledocolitiasis en pacientes con colelitiasis-colecistitis en Medellín.

Material y métodos. Se revisaron de manera retrospectiva 2.I22 historias clínicas de pacientes con diagnóstico de colelitiasis-colecistitis en el Hospital Pablo Tobón Uribe, de las cuales 20I fueron incluidas en este estudio. Se usó la escala propuesta de predicción de coledocolitiasis, evaluando los costos directos del modelo de uso de recursos diagnósticos.

Resultados. Se incluyeron 2OI pacientes (II7 mujeres y 84 hombres), con edad media de 54 años. El riesgo de coledocolitiasis en la población evaluada fue de $19,9 \%$, distribuido por grupos, así: población normal, o \%; con bajo riesgo, 5,26 \%; con riesgo intermedio, 52,6\%, y con riesgo alto, 93,7\%. El área bajo la curva ROC para el modelo predictivo fue de $0,95\left(\mathrm{IC}_{95 \%} \mathrm{O}, 92-0,98\right)$.

Según el análisis de los costos por grupos de riesgo, en los pacientes con riesgo normal, se obtuvo un sobrecosto de COP\$ 7'oro.0oo y, en aquellos con riesgo bajo, uno de COP\$44'013.500; en el grupo con riesgo medio, contrario a los demás grupos, se obtuvo una disminución de COP\$26’829.00o, mientras en el grupo de riesgo alto el sobrecosto fue de COP\$ 977.500.

Conclusiones. El modelo ofrece un buen rendimiento para predecir el riesgo de coledocolitiasis en la población estudiada. Si la escala se usa correctamente y se sigue el protocolo establecido con los pacientes, se disminuyen los estudios innecesarios y los costos, y se hace un uso racional de los recursos.

Palabras clave: conducto colédoco; coledocolitiasis; colelitiasis; colecistectomía laparoscópica; medición de riesgo; probabilidad.

Fecha de recibido: 23/08/2017 - Fecha aceptación: 16/02/2018

Correspondencia: Stevenson Orlando Londoño, Calle 36A Sur 26A-87, casa 104, Villas de Córcega, Medellín, Colombia / Teléfono: (300) 773-4561 / Correo electrónico: stevensonlondono@gmail.com

Citar como: Henao J, Londoño SO, Monsalve SC, Quintero VM, Sanabria AS. Validación externa del modelo HUSI para la predicción de coledocolitiasis en pacientes con colelitiasis-colecistitis. Rev Colomb Cir. 2018;33:135-44. https://doi.org/10.30944/20117582.55 


\begin{abstract}
Introduction. To determine the probability of choledocholithiasis in patients with cholelithiasis and to predict if the biliary stones are located at the common bile duct is a challenge for surgeons. Decision making can increase costs, so it is necessary to define the utility of predictive scores in these cases.

Objective. To validate the score to predict coledocolitiasis in patients with cholelithiasis developed by Domínguez, et al., in an external cohort in Medellín.

Material y methods. 2122 clinical records of patients with diagnosis of cholecystitis/ cholelithiasis were reviewed at the Hospital Pablo Tobón Uribe. The Dominguez score was used to predict probability of choledocholithiasis and we calculated the use of resources and costs. Area under ROC curve was calculated.

Results. 20I patients (II7 women and 84 men), with a mean age of 54 years were included. The frequency of choledocholithiasis was $19,9 \%$, distributed by risk groups: no risk, o \%; low risk, 5,26 \%; intermediate risk, 52,6 \%, and high risk, 93,7\%. Area under ROC curve was 0,95 $\left(\mathrm{IC}_{95 \%}\right.$ 0,92-0,98). Regarding costs, overrun was: COP\$ 7 '0I0.00o in patients without risk, COP\$ 44 '013.500 in those with low risk and COP\$ 977.500 in the high-risk group. In the intermediate risk group there was a saving of COP\$ $26^{\prime} 829.000$.

Conclusions. The Dominguez score offer good performance to predict choledocholithiasis. If the score is used according to recommendations, unnecessary costs decrease and more rational use of resources is achieved.
\end{abstract}

Key words: common bile duct; choledocholithiasis; cholelithiasis; cholecystectomy, laparoscopic; risk assessment; probability.

\section{Introducción}

La colelitiasis es una de las condiciones médicas más comunes que requieren intervención quirúrgica, y afecta aproximadamente al $\mathrm{I}_{5} \%$ de la población adulta en los Estados Unidos ${ }^{\mathrm{I}}$. La coledocolitiasis se desarrolla, aproximadamente, en el Io a $20 \%$ de los pacientes con colelitiasis y la literatura sugiere que, por lo menos, 3 a Io \% de los sometidos a colecistectomía tendrán coledocolitiasis ${ }^{\mathrm{I}}$. Entre el 21 y el $34 \%$ de los cálculos de la vía biliar principal migran espontáneamente al duodeno, y su migración entraña un riesgo (25-36 $\%$ ) de pancreatitis y colangitis, en caso de que se impacte en la vía biliar distal. En Colombia, se estima en II,9\% la frecuencia de la coledocolitiasis ${ }^{2}$.

En el $90 \%$ de los casos, la obstrucción por coledocolitiasis es incompleta y produce manifestaciones clínicas de obstrucción intermitente al paso de la bilis, en forma aguda o crónica. La mayoría de ellas son secundarias al paso de los cálculos de la vesícula biliar al colédoco.

La coledocolitiasis se puede diagnosticar en cualquier momento de la enfermedad y para ello se cuenta con varias ayudas diagnósticas, como los exámenes de laboratorio, imágenes como la ecografía hepatobiliar, la tomografía computa- dorizada (TC) y la resonancia magnética (RM), y métodos invasivos como la colangiografía intraoperatoria, la colangiopancreatografía retrógrada endoscópica (CPRE) y la ecoendoscopia ${ }^{3}$.

Con la transición de la colecistectomía abierta a los abordajes laparoscópicos, se intensificó el interés en los predictores preoperatorios de coledocolitiasis, pues la extracción de los cálculos de la vía biliar que se hacía directamente durante la colecistectomía abierta, ahora es técnicamente más complejo con la laparoscopia y el cirujano general está poco familiarizado con la técnica.

Las pruebas bioquímicas son el método menos invasivo que se ha evaluado para determinar la probabilidad de cálculos en los conductos biliares: el aumento de los niveles de alaninoaminotransferasa (ALT), aspartato-aminotransferasa (AST), bilirrubina y fosfatasa alcalina, se correlacionan con la presencia de cálculos en el conducto biliar y, si estos no están elevados, la probabilidad de coledocolitiasis es menor de $3 \%$ 4. Sin embargo, la sensibilidad y la especificidad individual de estas variables de laboratorio son insuficientes y varios grupos han tratado de identificar combinaciones de pruebas bioquímicas que tengan un valor predictivo superior. 
En el estudio de Barkun, et al. ${ }^{5}$, se demostró que la edad mayor de 55 años, una bilirrubinemia por encima de $30 \mathrm{mmol} / \mathrm{L}(\mathrm{I}, 8 \mathrm{mg} / \mathrm{dl})$ y una dilatación de más de $6 \mathrm{~mm}$ de la vía biliar superior, predijeron adecuadamente la presencia de cálculos en el colédoco, con una probabilidad del $92 \%$, siempre y cuando todos los valores de laboratorio estuvieran elevados. Sin embargo, en pacientes que se cree que tienen una gran probabilidad de cálculos de la vía biliar, con base en la dilatación del conducto en la ecografía y las pruebas hepáticas, se ha encontrado que no hay cálculos en el conducto biliar en más de las dos terceras partes de los casos ${ }^{6}$.

En razón de lo anterior, se han ideado reglas de predicción clínica para hacer el diagnóstico más certero o escoger la mejor opción diagnóstica y terapéutica en el enfoque del paciente con sospecha de coledocolitiasis. A pesar de los múltiples estudios que exploran el riesgo de coledocolitiasis en casos de colelitiasis, todavía sigue siendo un reto para el cirujano saber si realmente un cálculo se encuentra en la vía biliar. Además, el estudio de estos pacientes genera costos importantes para el sistema de salud.

Para el tratamiento de la coledocolitiasis, el principio consiste en eliminar el cálculo que produce la obstrucción de la vía biliar común mediante métodos que incluyen la CPRE, la exploración de la vía biliar abierta o laparoscópica y procedimientos percutáneos, simultáneamente con la colecistectomía laparoscópica. Aunque la CPRE es el enfoque menos invasivo para eliminar los cálculos del conducto biliar, se asocia con complicaciones en el $7 \%$ de pacientes 7 . Por lo tanto, es crítico que la CPRE se utilice solo para el tratamiento y no para el diagnóstico, lo que requiere evaluar con exactitud la probabilidad de cálculos en la vía biliar.

Domínguez, et al., diseñaron en el Hospital Universitario de San Ignacio (HUSI) ${ }^{2}$, un modelo de predicción a partir de la evidencia científica disponible, la experiencia local y la disponibilidad de recursos diagnósticos, con el fin de garantizar el uso racional de los recursos. Este se comparó con cinco modelos de predicción dispo- nibles en la literatura científica y se encontró que, con la escala, se demuestra un impacto directo en el uso de recursos según diferentes umbrales y ofrece mejor discriminación que otros, permitiendo el uso racional y eficiente de los recursos diagnósticos (tabla I).

El objetivo de este proyecto fue la validación externa del modelo descrito por Domínguez, et al., para determinar el riesgo de coledocolitiasis en el periodo preoperatorio en casos de colelitiasis, para decidir la conducta y conocer su impacto en el uso de recursos diagnósticos.

\section{Materiales y métodos}

Este fue un estudio de cohorte histórica. Como era un estudio observacional, considerado sin riesgo según la Resolución o08430 de 1993 del Ministerio de Salud de Colombia, no requirió de consentimiento informado y fue autorizado por la Oficina de Investigación y Docencia del Hospital Pablo Tobón Uribe.

La hipótesis planteada fue que, al tomar una decisión terapéutica basada en el puntaje del presente modelo predictivo para coledocolitiasis, se mantienen la misma discriminación y capacidad de predicción del modelo original, y se disminuye

Tabla 1. Escala de evaluación de coledocolitiasis en pacientes con colelitiasis, según Domínguez, et al. ${ }^{2}$

\begin{tabular}{lc}
\hline Factor & Puntos \\
\hline Vía biliar $>7 \mathrm{~mm}$ & 4 \\
Ictericia & 3 \\
ALT, 1,5 veces & 3 \\
Bilirrubina total >2 mg/dl & 2 \\
AST, 1,5 veces & 1 \\
Fosfatasa alcalina positiva & 1 \\
Pancreatitis & 1 \\
Edad<55 años & 1 \\
Total & 16 \\
\hline
\end{tabular}

Puntaje: 0 , riesgo normal de coledocolitiasis (o,8 \%); $\mathrm{I}-7$, riesgo bajo $(6,5 \%) ; 8$-I2, riesgo intermedio $(32 \%)$; I3-I6, riesgo alto $(59,3 \%)$ 
el número de estudios adicionales para hacer el diagnóstico de coledocolitiasis, en comparación con el manejo rutinario de la coledocolitiasis.

La población de estudio correspondió a pacientes adultos con dolor abdominal en el hipocondrio derecho y cuadro clínico sugestivo de colecistitis-colelitiasis, que consultaron a un centro de tercer nivel y que cumplieron con los siguientes criterios de inclusión: ser mayor de i8 años de edad y presentar colelitiasis con colecistitis o sin ella en cualquier estudio abdominal de imagen. Se excluyeron los pacientes: remitidos de otros hospitales con diagnóstico de coledocolitiasis confirmado por imágenes; con historia de pancreatitis de más de 72 horas antes de la hospitalización; con diagnóstico previo y confirmado de neoplasia periampular; con derivación previa de la vía biliar; con abdomen agudo y signos generalizados de irritación peritoneal; las mujeres embarazadas y aquellos con datos incompletos.

Se calculó un tamaño de la muestra con un error alfa de 0,05 , un beta de 0,2 , con una proporción del grupo control de $33 \%$ de uso de CPRE en el riesgo intermedio y una disminución esperada del $30 \%$ con el nuevo modelo, con lo cual el número de sujetos necesarios fue de 212. El muestreo fue no probabilístico de selección consecutiva.

Se seleccionaron y revisaron las historias de los pacientes que ingresaron al servicio de urgencias del Hospital Pablo Tobón Uribe y en quienes los cirujanos generales hicieron diagnóstico de colelitiasis-colecistitis. En estos pacientes se usó el modelo de predicción de coledocolitiasis diseñado por Domínguez, et al., mediante un instrumento prediseñado. Para clasificar el riesgo de coledocolitiasis, y con los datos obtenidos durante la atención inicial, se evaluaron la conducta clínica tomada con cada paciente (cirugía, colangiorresonancia o CPRE) y la conducta ideal según el modelo, y se comparó la frecuencia de coledocolitiasis y el uso de recursos diagnósticos según cada grupo de riesgo.

A cada recurso diagnóstico se le asignó un valor estándar de acuerdo con los precios del mercado. En cada grupo de riesgo, se calcularon el gasto real según la conducta clínica tomada y el gasto ideal siguiendo las recomendaciones propuestas por Domínguez, et al., las que eran: no usar ningún método diagnóstico diferente al provisto para la escala en casos de riesgo muy bajo, practicar la colangiografía intraoperatoria a liberalidad en casos de riesgo bajo, y practicar colangiorresonancia en casos de riesgo medio y CPRE en los de riesgo alto.

Los datos se registraron en una hoja de Excel (Microsoft) previamente adecuada para este uso.

Las variables categóricas se presentan en frecuencias, y las continuas, en promedio y desviación estándar para aquellas con distribución normal, y en mediana y rango intercuartílico para aquellas con distribución no normal. La normalidad fue evaluada con el método de Shapiro-Wilks. Las variables categóricas se compararon con la prueba de ji al cuadrado y, las variables continuas, con la prueba de t de Student. El modelo se calibró usando la curva ROC. La discriminación del instrumento se valoró según el riesgo de coledocolitiasis por categorías de la escala (baja, intermedia y alta), siguiendo las recomendaciones de validación externa de un modelo de predicción clínica descritas por Steyerberg, et al. ${ }^{8}$.

\section{Resultados}

Las historias fueron recolectadas en el Hospital Pablo Tobón Uribe, institución de alto nivel de complejidad ubicada en Medellín, Colombia, desde marzo de 2013 hasta enero de 2016.

Se evaluaron 2.122 historias clínicas de pacientes con diagnóstico de enfermedad biliar, de los cuales $20 \mathrm{I}$ cumplieron con los criterios de inclusión $(9,4 \%)$. En un análisis post hoc se demostró que el tamaño de la muestra de 20 r era suficiente para la hipótesis planteada y los hallazgos, por lo cual se suspendió el reclutamiento. El 58,2 \% de la población fueron mujeres y la edad media fue de 54 años. La frecuencia global de coledocolitiasis fue de $19,9 \%$. En la tabla 2, se muestran las características de la población evaluada.

Los límites para la clasificación de la escala fueron los mismos utilizados en el trabajo original de Domínguez, et al.: bilirrubina total mayor 
Tabla 2. Características de los pacientes

\begin{tabular}{|c|c|}
\hline Características & $\begin{array}{l}\text { Total de pacientes } \\
\qquad(\mathrm{N}=\mathbf{2 0 1})\end{array}$ \\
\hline Edad en años, media \pm DE & $54 \pm 18,8(19-89)$ \\
\hline Edad >55 años, n (\%) & $95 / 201(47,26 \%)$ \\
\hline Sexo: mujeres, n (\%) & $117 / 201(58,2 \%)$ \\
\hline Ictericia, n (\%) & $31 / 201(15,4 \%)$ \\
\hline Bilirrubina $(\mathrm{mg} / \mathrm{dl})$, media $\pm \mathrm{DE}$ & $1,74 \pm 2,12(0,26-9,4)$ \\
\hline Bilirrubina total >2,0 mg/dl, $\mathrm{n}(\%)$ & $44 / 201(21,8 \%)$ \\
\hline Fosfatasa alcalina, media $\pm \mathrm{DE}$ & $155,24 \pm 146,93(34-599)$ \\
\hline Fosfatasa alcalina positiva, n (\%) & $55 / 201(27,36 \%)$ \\
\hline $\mathrm{AST}$, media $\pm \mathrm{DE}$ & $121,05 \pm 241,46(10-1.103)$ \\
\hline AST > 1,5, n (\%) & $76 / 201(37,8 \%)$ \\
\hline $\mathrm{ALT}$, media $\pm \mathrm{DE}$ & $137,41 \pm 251,83(9-1.356)$ \\
\hline $\mathrm{ALT}>1,5, \mathrm{n}(\%)$ & $63 / 201(31,34 \%)$ \\
\hline Pancreatitis, n (\%) & 28/201 (13,93\%) \\
\hline $\begin{array}{l}\text { Diámetro del colédoco }(\mathrm{mm}) \\
\text { media } \pm \mathrm{DE}\end{array}$ & $6,0 \pm 2,87(4-17)$ \\
\hline Diámetro del colédoco >7 mm, $\mathrm{n}(\%)$ & $41(20,4 \%)$ \\
\hline Muerte & $2(1 \%)$ \\
\hline
\end{tabular}

de $2,0 \mathrm{mg} / \mathrm{dl}$, ALT y AST en I,5 veces su valor normal (normal de o a 55 y de 5 a 34 UI, respectivamente), fosfatasa alcalina elevada (normal de 40 a I50 UI, y diámetro del colédoco mayor de 7 $\mathrm{mm}$. Con estos datos, se construyó un puntaje sumando: 3 puntos por ictericia, más I por pancreatitis, más 4 por diámetro del colédoco $>7 \mathrm{~mm}$, más 3 por bilirrubina total $>2 \mathrm{mg} / \mathrm{dl}$, más I por fosfatasa alcalina $>$ I26, más I por AST $>90$, más 3 por ALT>IO8, con o a I6 como un valor probable ${ }^{2}$.

$\mathrm{El}$ área bajo la curva ROC para el modelo predictivo, fue de 0,95 $\left(\mathrm{IC}_{95 \%}\right.$ 0,92-0,98) (figura I).

Según los resultados de la escala, los pacientes se agruparon en las cuatro categorías establecidas por el modelo de predicción, con una frecuencia de coledocolitiasis así: puntaje o, normal (riesgo de coledocolitiasis o \%); puntaje $\mathrm{I}-5$, riesgo bajo $(5,26 \%)$, puntaje $6-\mathrm{I} 2$, riesgo intermedio (52,6\%); y puntaje I3-I6, riesgo alto (93,7\%) (tabla 3).

Para calcular los costos se usaron los siguientes valores de los recursos diagnósticos: $\mathrm{TC}$ de $\mathrm{ab}$ domen, COP\$ I'I43.00o, RM de abdomen, COP\$ I'650.000, CPRE diagnóstica, COP\$ 2'500.000, y CPRE instrumentada, COP\$ 5'000.00o. En las tablas 4 a 6, se muestra la distribución del uso de recursos diagnósticos por riesgo de coledocolitiasis según el modelo, en la tabla 7, el tiempo de hospitalización según el riesgo, y en la tabla 8 , el cálculo total de costos.

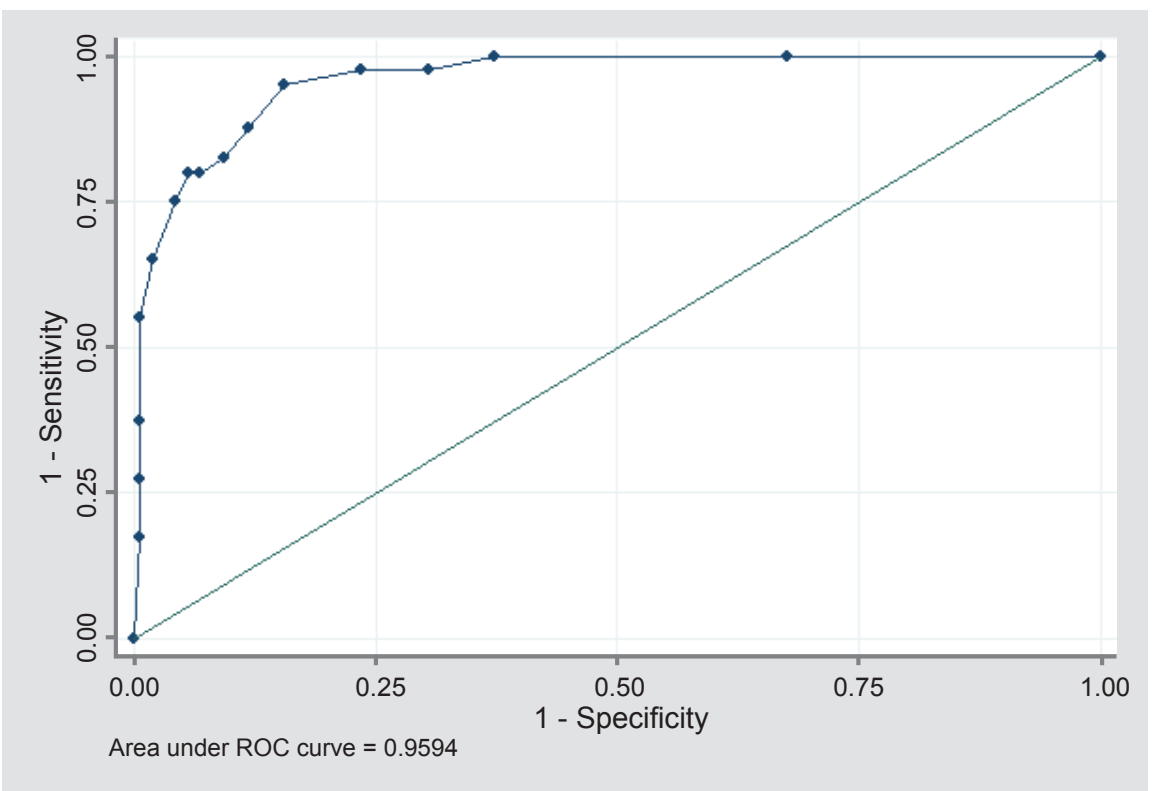

Figura 1. Curva ROC para valorar la calibración de la escala HUSI. 
Tabla 3. Riesgo de coledocolitiasis según el grupo de riesgo de la escala

\begin{tabular}{lcccc}
\hline \multicolumn{1}{c}{ Riesgo } & Pacientes & $\begin{array}{c}\text { Coledocolitiasis } \\
\mathbf{n}(\%)\end{array}$ & $\begin{array}{c}\text { Coledocolitiasis según la } \\
\text { escala original de Domínguez, } \\
\text { et al. }{ }^{2}(\%)\end{array}$ \\
\hline Normal (0) & 52 & 25,87 & $0(0)$ & $0(0)$ \\
Bajo (1) & 95 & 47,26 & $5(5,3)$ & $4(7)$ \\
Medio (2) & 38 & 18,91 & $20(52,6)$ & $17(29,8)$ \\
Alto (3) & 16 & 7,96 & $15(93,7)$ & $6(50)$ \\
Total & 201 & 100 & $40(19,9)$ & $27(14,4)$ \\
\hline
\end{tabular}

Tabla 4. Uso de la CPRE y éxito por grupo de riesgo

\begin{tabular}{lcccc}
\hline \multicolumn{1}{c}{ Riesgo } & $\begin{array}{c}\text { Pacientes } \\
\mathbf{n}\end{array}$ & $\begin{array}{c}\text { Coledocolitiasis } \\
\mathbf{n}(\%)\end{array}$ & $\begin{array}{c}\text { CPRE practicadas } \\
\mathbf{n}\end{array}$ & $\begin{array}{c}\text { CPRE exitosa } \\
\mathbf{n}(\%)\end{array}$ \\
\hline Normal $(0)$ & 52 & 0 & 0 & 0 \\
Bajo (1) & 95 & $5(5,2)$ & 6 & $5(83,3)$ \\
Medio (2) & 38 & $20(52,63)$ & 20 & $12(60)$ \\
Alto (3) & 16 & $15(93,75)$ & 15 & 11 \\
Total & 201 & 40 & $41^{*}$ & 28 \\
\hline
\end{tabular}

*Un paciente requirió dos CPRE.

Tabla 5. Uso de TC por grupo de riesgo

\begin{tabular}{lccc}
\hline Riesgo & $\begin{array}{c}\text { Pacientes } \\
\mathbf{n}\end{array}$ & $\begin{array}{c}\text { Coledocolitiasis } \\
\mathbf{n}\end{array}$ & $\begin{array}{c}\text { TC practicadas } \\
\mathbf{n}(\%)\end{array}$ \\
\hline Normal (0) & 52 & 0 & $3(9)$ \\
Bajo (1) & 95 & 5 & $22(65)$ \\
Medio (2) & 38 & 20 & $5(15)$ \\
Alto (3) & 16 & 15 & $4(12)$ \\
Total & 201 & 40 & $34(100)$
\end{tabular}

*Comparación entre el número de TC del modelo original y el de la cohorte de validación

Tabla 6. Uso de RM por grupo de riesgo

\begin{tabular}{lccc}
\hline Riesgo & $\begin{array}{c}\text { Pacientes } \\
\mathbf{n}\end{array}$ & $\begin{array}{c}\text { Coledocolitiasis } \\
\mathbf{n}\end{array}$ & $\begin{array}{c}\text { RM } \\
\text { practicadas } \\
\mathbf{n}(\%)\end{array}$ \\
\hline Normal (0) & 52 & 0 & $1(3)$ \\
Bajo (1) & 95 & 5 & $10(32)$ \\
Medio (2) & 38 & 20 & $17(55)$ \\
Alto (3) & 16 & 15 & $3(10)$ \\
Total & 201 & 40 & 31 \\
\hline
\end{tabular}

*Comparación entre el número de RM del modelo original y el de la cohorte de validación

Tabla 7. Días de hospitalización por grupo de riesgo

\begin{tabular}{lc}
\hline Riesgo & Media (rango) \pm DE \\
\hline Normal (0) & $2,0 \pm 1,2(1-5)$ \\
Bajo (1) & $6,7 \pm 10,1(0-64)$ \\
Medio (2) & $7,8 \pm 5(1-24)$ \\
Alto (3) & $10 \pm 7,3(2-28)$ \\
\hline
\end{tabular}

Tabla 8. Tabla de gastos por grupo de riesgo

\begin{tabular}{|c|c|c|c|c|c|c|c|c|c|c|c|c|c|}
\hline $\begin{array}{l}\text { Nivel de } \\
\text { riesgo }\end{array}$ & $\begin{array}{c}\text { Número } \\
\text { de } \\
\text { pacientes }\end{array}$ & $\begin{array}{l}\text { Número de } \\
\text { pacientes } \\
\text { con coledo- } \\
\text { colitiasis }\end{array}$ & $\begin{array}{c}\% \\
\text { CPRE }\end{array}$ & $\begin{array}{l}\text { Valor } \\
\text { CPRE }\end{array}$ & $\%$ TC & Valor TC & $\%$ RM & Valor RM & $\begin{array}{c}\text { Valor } \\
\text { laboratorios }\end{array}$ & $\begin{array}{c}\text { Exceso de } \\
\text { estancia } \\
\text { (días) }\end{array}$ & $\begin{array}{c}\text { Valor } \\
\text { estancia }\end{array}$ & Valor ideal & $\begin{array}{c}\text { Gasto } \\
\text { innecesario }\end{array}$ \\
\hline 0 & 52 & 0 & $0,0 \%$ & $\$-$ & $5,7 \%$ & $\$ 3.429 .000$ & $1,9 \%$ & $\$ 1.650 .000$ & $\$ 1.931 .000$ & 0 & $\$-$ & $\$-$ & $\$ 7.010 .000$ \\
\hline 1 & 95 & 5 & $6,0 \%$ & $\$ 15.000 .000$ & $23,1 \%$ & $\begin{array}{r}\$ \\
25.146 .000\end{array}$ & $10,5 \%$ & $\$ 16.500 .000$ & $\$ 4.226 .500$ & 2 & $\$ 451.000$ & $\$ 17.310 .000$ & $\$ 44.013 .500$ \\
\hline 2 & 38 & 20 & $52,6 \%$ & $\$ 50.000 .000$ & $13,1 \%$ & $\$ 5.715 .000$ & $44,7 \%$ & $\$ 28.050 .000$ & $\$ 1.880 .500$ & 1 & $\$ 225.500$ & $\$ 162.700 .000$ & $-\$ 26.829 .000$ \\
\hline 3 & 16 & 15 & $93,7 \%$ & $\$ 37.500 .000$ & $25,0 \%$ & $\$ 4.572 .000$ & $18,7 \%$ & $\$ 4.950 .000$ & $\$ 817.500$ & 6 & $\$ 1.353 .000$ & $\$ 85.715 .000$ & $\$ 977.500$ \\
\hline Total & & & & & & & & & & & & & $\$ 25.172 .000,00$ \\
\hline
\end{tabular}

CPRE: colangiopancreatografía retrógrada endoscópica; TC: tomografía computadorizada; RM: resonancia magnética 


\section{Discusión}

A pesar de los múltiples estudios sobre la determinación de la probabilidad de coledocolitiasis que tienen los pacientes con colelitiasis, todavía sigue siendo un reto para el cirujano saber si realmente un cálculo se encuentra en la vía biliar y cuál debe ser la prueba para esclarecer el diagnóstico. Estas decisiones se ven reflejadas en costos para el sistema de salud.

En 20II, Domínguez, et al., ${ }^{2}$ desarrollaron un modelo de predicción para discriminar el riesgo de coledocolitiasis, el cual intenta hacer un uso racional de los recursos diagnósticos de imágenes y pruebas bioquímicas de rutina, en este tipo de pacientes. En este modelo, mostró un área adecuada bajo la curva ROC, con optimización del recurso en pacientes de riesgo medio. Una de sus debilidades fue que no es válido en una población diferente, lo que conlleva riesgo de sobreestimación del modelo. Por esta razón, se decidió validar el modelo de predicción en una población y un lugar geográfico diferentes, para determinar su desempeño de acuerdo con las recomendaciones de la iniciativa TRIPOD (Transparent Reporting of a multivariable prediction model for Individual Prognosis Or Diagnosis) 9.

En este estudio, se evaluaron 2.I22 historias clínicas de pacientes con diagnóstico de enfermedad biliar, de los cuales solo 20 I cumplieron con los criterios de inclusión. Se estudiaron todos los que fueron admitidos con sospecha de enfermedad biliar en la institución, en el periodo de estudio.

La mayoría de los excluidos presentaban: datos incompletos en el perfil bioquímico o en los resultados de la ecografía, ausencia de este examen o enfermedad biliar maligna. También, se excluyeron aquellos derivados a otras instituciones por razones administrativas por su entidad prestadora de salud (EPS), lo cual fue causa de la gran mayoría de las pérdidas.

Solo se incluyó el $9 \%$ de los potenciales candidatos. Infortunadamente, la pérdida de pacientes por falta de pruebas bioquímicas o imaginológicas para el abordaje de la enfermedad biliar, evidenció falta de universalidad en el estudio de la enfermedad biliar obstructiva y de familiaridad con las escalas de riesgo, lo que hace que los pacientes sean programados para cirugía sin establecer adecuadamente el riesgo de coledocolitiasis y sin tener los estudios previos requeridos para la colecistectomía.

Cuando se usó la escala, se encontró que el riesgo global de coledocolitiasis en esta población fue de $19,9 \%$, cifra que es similar a la de diferentes series publicadas, las cuales establecen un riesgo de 5 a $20 \%$ 3,4,ro,II. La mayoría de los modelos publicados muestran un buen desempeño ${ }^{12-17}$, pero los recursos humanos y tecnológicos disponibles, la experiencia de los cirujanos y la prevalencia de coledocolitiasis, hacen difícil su aplicación universal.

Las características del grupo poblacional que se encontró durante esta revisión, son comparables con las que describió Barkun, et al., aunque se encontró una prevalencia un poco mayor en el sexo femenino ${ }^{5}$. El I5,4 \% de los pacientes presentaba ictericia clínica y, el 2I,8\%, valores de bilirrubina por encima de 2 , lo que es comparable con la población que dichos autores describieron desde 1994 y que, en la actualidad, sugieren las recomendaciones de la Society of American Gastrointestinal and Endoscopic Surgeons (SAGES) ${ }^{12}$.

La curva ROC del modelo predictor constituye un método estadístico para determinar la calibración diagnóstica del modelo. Esta mostró una adecuada calibración, lo cual ayuda a distinguir entre los pacientes sanos y enfermos $(\mathrm{ROC}=0,95)\left(\mathrm{IC}_{95 \%}\right.$ 0,92-0,98), lo que demuestra que se puede utilizar en nuestra población. Los resultados son mejores que los reportados por Domínguez, et al. ${ }^{2}$, con un área bajo la curva de 0,88 , Houdart, et al. ${ }^{13}$, con una de 0,66 , y Stain, et al. ${ }^{14}$, con una de 0,62 .

El modelo predictivo en esta población reportó un riesgo de coledocolitiasis de 5,26\% para el grupo de riesgo bajo, de 52,6 \% para el de riesgo intermedio y de $93,7 \%$ para el de riesgo alto, lo cual muestra un adecuado poder discriminatorio en comparación con lo descrito en la escala original $^{2}$, pero mucho mejor que los mostrados en los tres estudios anteriores. 
De los pacientes que se encontraron sin riesgo $(25,9 \%)$, equivalente al puntaje o en la escala, ninguno presentó coledocolitiasis, lo que indica que estos no se beneficiarían de ningún otro método diagnóstico adicional diferente a la colecistectomía. En este grupo, hubo una estancia hospitalaria media de dos días, que podría acortarse practicando la cirugía de forma ambulatoria. Cuando no se usó el modelo, aumentaron los días de estancia hospitalaria, el uso de ayudas diagnósticas y el de otros procedimientos, lo cual, a su vez, incrementó el costo de la atención.

De los pacientes con riesgo bajo (47,26\%), tan solo el 5,26\% presentó coledocolitiasis, lo cual concuerda con los resultados reportados en otros estudios ${ }^{2,1,1,1,2,18,19}$. La media de hospitalización fue de 6,7 días para esta población, la cual consideramos alta, ya que estos pacientes no requieren ayudas diagnósticas diferentes a las rutinarias. El uso inadecuado de métodos diagnósticos retrasa la atención del paciente y el tratamiento de la enfermedad. En estos casos, basta con practicar una colecistectomía laparoscópica, una colangiografía in traoperatoria o ambas, según los hallazgos quirúrgicos, como se recomienda en algunas guías ${ }^{15}$. Infortunadamente, en nuestro medio, el cirujano no está familiarizado con la colangiografía intraoperatoria, en muchos de los quirófanos no se dispone del recurso de manera rápida y las imágenes no son leídas de forma oportuna por el especialista, lo cual puede conllevar una mala interpretación.

En el grupo con riesgo intermedio, la frecuencia de coledocolitiasis fue del 52,6\%, mayor que la reportada por Domínguez, et al. ${ }^{2}$. Esta mejoría en la discriminación probablemente obedece a la incorporación progresiva de las variables descritas en el modelo original y que cada vez más son tomadas en cuenta por los cirujanos como variables independientes en la toma de decisiones, aun fuera del uso formal de la escala. Está claro que la población de este grupo debe estudiarse para descartar coledocolitiasis. El método de elección propuesto por Domínguez, et al. ${ }^{2}$, fue la RM dadas sus características operativas. No obstante, muchos de estos pacientes se es- tudiaron inicialmente con TC de abdomen o se sometieron directamente a la CPRE. $\mathrm{Al} 34 \%$ se les practicaron estudios innecesarios, lo cual aumenta los riesgos inherentes a la CPRE en una población que no requiere de este método diagnóstico como primera elección. $\mathrm{Al}$ I5 \% se le practicó TC de abdomen, lo que implica un uso inadecuado de los recursos, y retraso en el diagnóstico y el tratamiento de la enfermedad.

En la cohorte actual, los resultados mostraron que hubo un uso menor del esperado de estos métodos diagnósticos sin el uso de la escala y se gastaron menos recursos. No se encontró explicación para este comportamiento, pero se piensa que puede deberse al acatamiento de las recomendaciones de la SAGES (Society of American Gastrointestinal and Endoscopic Surgeons) que fueron introducidas en la práctica clínica en los últimos años y que son muy similares a la propuesta original de Domínguez, et al. ${ }^{2}$.

La TC se usó en I2 \% y la RM en Io \% de los integrantes del grupo de riesgo alto $(7,9 \%)$. En estos pacientes, dichos exámenes hubieran podido evitarse y la remisión para practicarles una CPRE terapéutica podría haber sido más oportuna. La estancia media hospitalaria fue de Io días y hubiera sido menor si se hubiera optado por el uso temprano de la CPRE.

Es claro en la literatura científica que la sensibilidad de la TC no es muy lejana a la de la ecografía para detectar la coledocolitiasis (86 Vs. $82 \%)$ y la especificidad es similar $(98 \%)^{14}$. En este estudio se encontró que, de todas las TC de abdomen practicadas en los diferentes grupos de riesgo, el $9 \%$ se realizó en pacientes con riesgo o (normal) y el $65 \%$ en los de riesgo bajo, para un total de $74 \%$ de TC en una población que no requiere inicialmente ningún examen de imagen adicional a la ecografía. Además, en esta misma población se practicó el $35 \%$ de las RM de abdomen. Todo esto conlleva mayor estancia hospitalaria, aumento de los costos de la atención en salud y retraso para el manejo oportuno de la enfermedad.

El preferir la TC en lugar de la RM cuando se sospecha coledocolitiasis, cuando esta última 
ha mostrado ser mucho mejor en la enfermedad biliar, denota falta de familiaridad con métodos diagnósticos que le permiten al clínico tomar una buena decisión durante el proceso de atención y falta de un diagnóstico clínico preciso que lleve a solucionar la enfermedad del paciente. El solicitar una TC en toda enfermedad abdominal se ha convertido en una costumbre para muchos cirujanos, ya sea porque se practica más rápido que la RM, porque el asegurador se demora menos en autorizarla, por su mayor disponibilidad o porque el médico se encuentra más familiarizado con sus imágenes.

La CPRE es el método de referencia para el tratamiento de la coledocolitiasis. En la década de los 9o, la Society of American Gastrointestinal and Endoscopic Surgeons declaró, que un endoscopista debe ser capaz de alcanzar, como mínimo, una tasa de éxito del $85 \%$ en los procedimientos más comunes, como la extracción de cálculos de la vía biliar o el alivio de la obstrucción biliar ${ }^{15,16}$. La CPRE es un procedimiento que conlleva riesgos, $y$ complicaciones a corto y a largo plazo, por lo que se debe utilizar en casos seleccionados y con una indicación clara. La tasa de éxito en la extracción del cálculo fue del 68,2 \% y este éxito fue mayor en el grupo de alto riesgo (73,3\%), mientras que en los pacientes con riesgo intermedio fue de $60 \%$.

$\mathrm{Al}$ analizar el costo de los recursos utilizados en la población de estudio (tabla 8) se encontró que, para los pacientes clasificados como sin riesgo, se tuvo un sobrecosto, aproximadamente, de COP\$ 7’oro.ooo, debido al uso de exámenes de imágenes no pertinentes para esta población.

Para la población de bajo riesgo, se obtuvo un sobrecosto de COP\$ 44'0I3.500. Esta población tiene un riesgo de coledocolitiasis de $6 \% \mathrm{y}$, por lo tanto, este valor está ligado al uso de imágenes diagnósticas innecesarias.

Para la población en riesgo medio, contrario a los demás grupos, se obtuvo una disminución de COP\$ 26’829.000. Esto puede explicarse por otros factores, como la decisión precoz de practicar directamente la CPRE, sin previos exámenes de imágenes, en casos de cálculo en el colédoco reportado en la ecografía o valores muy elevados de bilirrubinas. Este aspecto no pudo determinarse en este estudio.

Por último, los pacientes con riesgo alto tuvieron un sobrecosto de COP\$977.500, lo cual se relacionado por el uso de imágenes diagnósticas innecesarias (como la TC) y, por ende, una estancia hospitalaria más prolongada. Cuando se analizan comparativamente los recursos económicos utilizados, el sobrecosto en la atención de esos pacientes ascendió a COP\$25' 172 .00o pesos.

Por ser retrospectivo, este estudio adolece de falencias y sesgos de información. A pesar de que la población inicial fue inmensa, la pérdida de pacientes potenciales fue importante. Por otra parte, el uso poco frecuente de este tipo de escala en nuestro medio hace que no se soliciten de manera completa los estudios requeridos. Otro factor es el efecto de la "contaminación" de la cohorte y la toma de decisiones con los datos del modelo previamente definido, y que en el transcurso de los años ha ido introduciéndose de manera informal en la toma de decisiones. Esto explica por qué la discriminación actual es mucho mejor que la definida originalmente.

Como recomendación, es necesario hacer un ensayo clínico aleatorizado y multicéntrico para comparar esta escala con otras actualmente utilizadas, para no sobreestimar la misma población y para predecir mejor su impacto en el consumo de los recursos.

\section{Conclusión}

La validación de este modelo de predicción, cuando se usó en una población de Medellín, ofrece una buena calibración a la hora de predecir el riesgo de coledocolitiasis. El uso adecuado de esta escala en los pacientes con colecistitiscolelitiasis, permite seguir un protocolo establecido y lleva a disminuir el uso de exámenes de imagen y otros innecesarios, a minimizar los costos y a usar racionalmente los recursos.

Creemos que se debe difundir el modelo en las instituciones prestadoras de salud y crear algoritmos de manejo en los sistemas institucionales, y que se deben buscar alternativas para 
su uso más amplio y cómodo, como el diseño de una aplicación para teléfonos celulares, buscando que esté a disposición de todos los médicos de urgencias. Consideramos que sería útil dar a conocer a los pacientes que existen estos métodos de predicción, pues pueden servir como una forma de control externo a la práctica quirúrgica cotidiana.

\section{Conflicto de interés ninguno.}

\section{Referencias}

I. Everhart JE, Khare M, Hill M, Maurer KR. Prevalence and ethnic differences in gallbladder disease in the United States. Gastroenterol. 1999;117:632-9.

2. Domínguez L, Niño N, Rubio O, Valdivieso E, Rugeles S, Sanabria Á. Desarrollo de un modelo para la predicción de coledocolitiasis: análisis e impacto de la discriminación de riesgo en el uso de recursos. Medicina. 20II;33:160-75.

3. Caddy GR, Tham TC. Symptoms, diagnosis and endoscopic management of common bile duct stones. Best Pract Res Clin Gastroenterol. 2006;20:I085-IIOI.

4. Ang TL, Teo EK, Fock KM. Endosonography Vs. endoscopic retrograde cholangiopancreatography-based strategies in the evaluation of suspected common bile duct stones in patients with normal transabdominal imaging. Aliment Pharmacol Ther. 2007;26:1163-70.

5. Barkun AN, Barkun JS, Fried GM, Ghitulescu G, Steinmetz O, Pham C, et al. Useful predictors of bile duct stones in patients undergoing laparoscopic cholecystectomy. McGill Gallstone Treatment Group. Ann Surg. 1994;220:32-9.

6. Sarli L, Costi R, Gobbi S, Sansebastiano G, Roncoroni L. Asymptomatic bile duct stones: Selection criteria for intravenous cholangiography and/or endoscopic retrograde cholangiography prior to laparoscopic cholecystectomy. Eu J Gastroenterol Hepatol. 2000;I2:II7580.

7. Andriulli A, Loperfido S, Napolitano G, Niro G, Valvano $\mathrm{MR}$, Spirito F, et al. Incidence rates of post-ERCP complications: A systematic survey of prospective studies. Am J Gastroenterol. 2007;102:I78I-8.

8. Steyerberg EW, Vickers AJ, Cook NR, Gerds T, Gonen $\mathrm{M}$, Obuchowski N, et al. Assessing the performance of prediction models: A framework for some traditional and novel measures. Epidemiology. 2010;21:128-38.

9. Localio AR, Stack CB. TRIPOD: A new reporting baseline for developing and interpreting prediction models. Ann Intern Med. 2015;162:73-4.

Io. Williams EJ, Green J, Beckingham I, Parks R, Martin D, Lombard M. Guidelines on the management of common bile duct stones (CBDS). Gut. 2008;57:1004-2I.

II. Onken JE, Brazer SR, Eisen GM, Williams DM, Bouras EP, DeLong ER, et al. Predicting the presence of choledocholithiasis in patients with symptomatic cholelithiasis. Am J Gastroenterol. 1996;9I:762-7.

I2. Maple JT, Ben-Menachem T, Anderson MA, Appalaneni V, Banerjee S, Cash B, et al. The role of endoscopy in the evaluation of suspected choledocholithiasis. Gastrointes Endosc. 20I0;7I:I-9.

I3. Houdart R, Perniceni T, Darne B, Salmeron M, Simon JF. Predicting common bile duct lithiasis: Determination and prospective validation of a model predicting low risk. Am J Surg. 1995;I70:38-43.

I4. Stain SC, Marsri LS, Froes ET, Sharma VISH, Parekh DILIP. Laparoscopic cholecystectomy: Laboratory predictors of choledocholithiasis. Am Surg. 1994;60:767-7I.

I5. Yamashita Y, Takada T, Kawarada Y, Nimura Y, Hirota M, Miura F, et al. Surgical treatment of patients with acute cholecystitis: Tokyo Guidelines. Journal of Hepato-biliary-pancreatic Surgery. 2007;I4:9I-7.

I6. Sgourakis G, Dedemadi G, Stamatelopoulos A, Leandros E, Voros D, Karaliotas K. Predictors of common bile duct lithiasis in laparoscopic era. World J Gastroenterol. 2005;II:3267-72.

I7. Sheen AJ, Asthana S, Al-Mukhtar A, Attia M, Toogood GJ. Preoperative determinants of common bile duct stones during laparoscopic cholecystectomy. J Clin Practice. 2008;62:1715-9.

I8. Byrne MF, McLoughlin MT, Mitchell RM, Gerke H, Kim K, Pappas TN, et al. For patients with predicted low risk for choledocholithiasis undergoing laparoscopic cholecystectomy, selective intraoperative cholangiography and postoperative endoscopic retrograde cholangiopancreatography is an effective strategy to limit unnecessary procedures. Surg Endosc. 2009;23:1933-7.

I9. Bose SM, Mazumdar A, Prakash SV, Kocher R, Katariya $\mathrm{S}$, Pathak CM. Evaluation of the predictors of choledocholithiasis: Comparative analysis of clinical, biochemical, radiological, radionuclear, and intraoperative parameters. Surg Today. 200I;31:II7-22. 